\title{
The perplexing case of the geranylgeranyl transferase-deficient mouse
}

\author{
Mark R. Philips \\ New York University Cancer Institute, New York, New York, USA.
}

\begin{abstract}
Proteins that end with a CAAX sequence are targeted to cellular membranes by a series of posttranslational modifications that include prenylation, proteolysis, and carboxyl methylation. Two prenyltransferases modify CAAX proteins: farnesyltransferase and geranylgeranyltransferase type I (GGTase-I). Rho family GTPases that control the actin cytoskeleton and are therefore critical to inflammatory cell function are substrates for GGTase-I. In this issue of the JCI, Khan et al. examined mice in which GGTase-I was conditionally deleted in macrophages. Rather than obtunded cells, the authors found activated Rho proteins in fully functional macrophages that hypersecreted inflammatory cytokines and induced an erosive, inflammatory arthritis. This surprising result calls into question the role of protein geranylgeranylation in inflammatory cell signaling.
\end{abstract}

The most interesting results in experimental biology are those that are unexpected. Never has this been truer than in the age of transgenic mice. Now that we can manipulate the rodent genome at will, we are frequently dumbfounded. H-Ras, the protagonist of decades of studies of cell signaling, turned out to be entirely dispensable (1). TGF- $\beta 1$, thought to be a growth factor, proved to be critically important to the immune system, where it protects against massive inflammatory infiltration of vital organs (2). We can now add to this list of perplexing results the data reported in this issue of the JCI by Khan et al. (3). These investigators tested the hypothesis that deficiency in macrophages of an enzyme that prenylates a variety of key signaling molecules would ameliorate inflammation. Instead, they found hyperactivated macrophages that secreted inflammatory cytokines and induced an autoimmune inflammatory arthritis resembling human rheumatoid arthritis.

\section{Protein prenylation and Rho GTPases}

To appreciate why the authors expected mice lacking geranylgeranyltransferase type I (GGTase-I) in macrophages to be protected from inflammatory disease, one must understand the fundamentals

Conflict of interest: The author has declared that no conflict of interest exists.

Citation for this article: J Clin Invest. 2011; 121(2):510-513. doi:10.1172/JCI45952. of posttranslational processing of the Ras superfamily of small GTPases. Mammalian genomes encode more than 150 small GTPases that operate as binary switches and control a wide range of cellular functions, including growth, differentiation, motility, and vesicular trafficking (4). Most small GTPases are constitutively and efficiently posttranslationally modified with lipids that are believed to target the proteins to the cellular membranes upon which they perform their various functions. The pathways through which these modifications occur are highly conserved from yeast to humans. Ras and Rho family GTPases terminate in a CAAX sequence that is modified by prenylation with one of two polyisoprene lipids: 15-carbon farnesyl or 20-carbon geranylgeranyl (5). When the amino acid in position $\mathrm{X}$ of the CAAX sequence is leucine, the protein is modified by GGTase-I, and a geranylgeranyl moiety is added to the CAAX cysteine via a stable thioether linkage. CAAX sequences that terminate with other amino acids are instead substrates for farnesyltransferase (FTase). Among the CAAX proteins that are substrates for GGTase-I are most Rho family proteins. Prenylation is considered essential for the biological function of small GTPases, and a great deal of in vitro experimental evidence supports this idea $(6,7)$.

The best-characterized function of Rho family GTPases is regulation of the actin cytoskeleton (8). In this way, Rho proteins control membrane protrusion and retrac- tion and all related functions, such as motility and phagocytosis. Accordingly, leukocytes are considered to be particularly beholden to Rho proteins for their various functions, and this has been borne out experimentally. For example, Rac2deficient leukocytes have a marked defect in chemotaxis (9). Thus, it is easy to understand why Khan et al. expected GGTase-Ideficient leukocytes to be defective and thereby limit inflammation (3).

\section{The mouse with GGTase-I-deficient macrophages}

FTase and GGTase-I are heterodimeric enzymes that combine a common $\alpha$ subunit with unique $\beta$ subunits. Khan et al. targeted the gene encoding the GGTase-I $\beta$ subunit, known as Pggt1b, to produce a conditional Pggt $1 b^{f l}$ allele (10). In previously published work using this allele, the authors showed that GGTase-I deficiency ameliorated K-Ras-driven cancer, suggesting that, as expected, K-Ras-mediated oncogenesis requires geranylgeranylation of Rho family proteins (10). In the present study, Khan et al. bred Pggt $1 b^{f l / f l}$ mice with mice that express Cre recombinase from a lysozyme $\mathrm{M}$ promoter that is active in myeloid cells, particularly macrophages (3). The authors then compared Pggt $1 b^{f l+} L C$ with $P g g t 1 b^{f l f f} L C$ mice. They confirmed efficient Cre-mediated excision of the floxed allele and showed that recombination was restricted to myeloid cells. Importantly, recombination was not observed in dendritic cells or lymphocytes. Tests for residual GGTase-I activity in $P g g t 1 b^{f l / f l} L C$ macrophages were negative. Thus, at the molecular level, the authors accomplished what was intended.

The surprises came with developmental, pathophysiological, and cell biological analysis of the mice $(3,10)$. Myelopoiesis was intact in $P g g t 1 b^{f / / f l} L C$ mice, which suggested that the complex process of differentiation from myeloid stem cells, which involves, among other things, production of specialized cytoplasmic vesicles, does not require GGTase-I $(3,10)$. Even more 


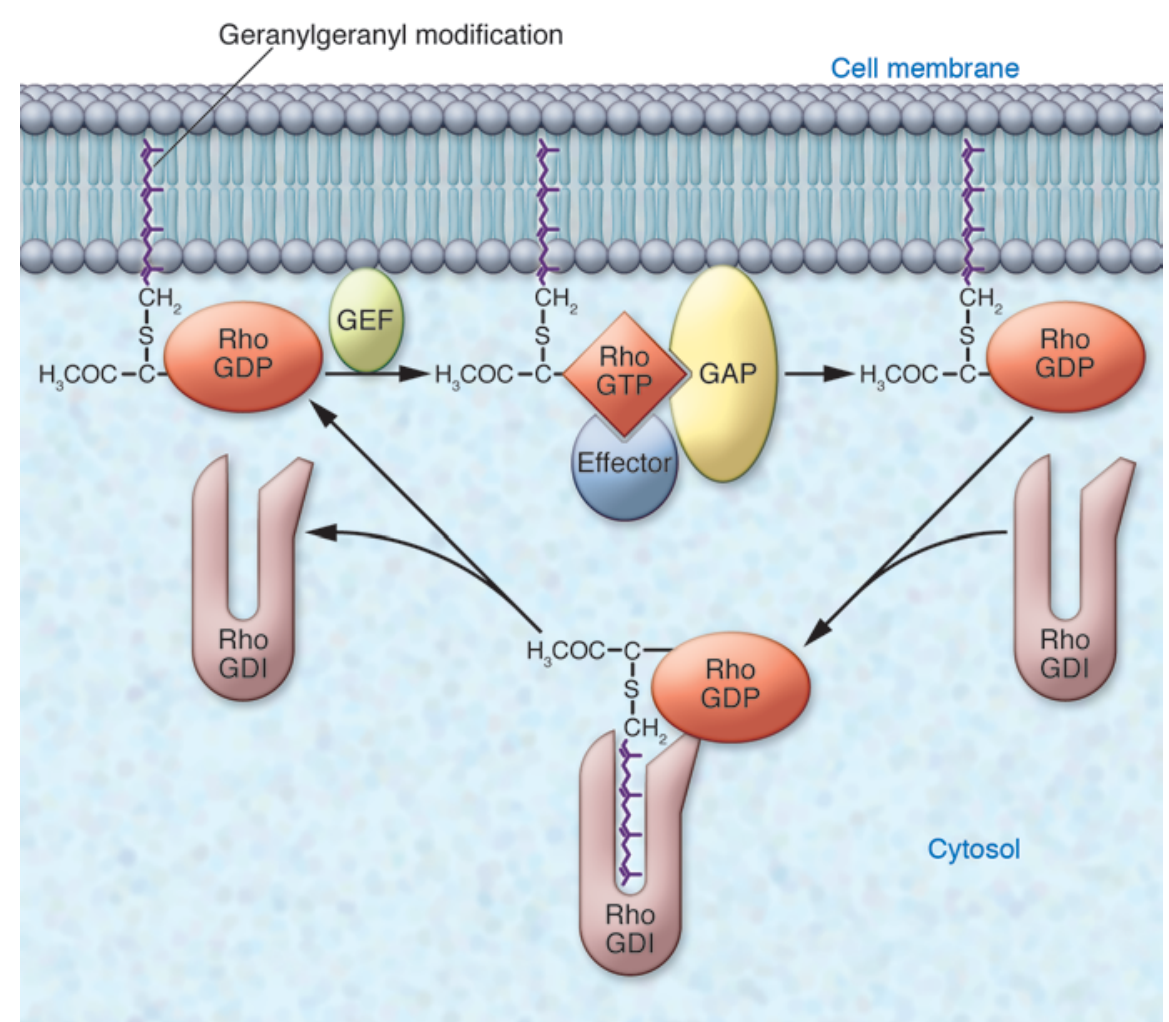

unexpected was the pathology: Pggt $1 b^{f l / f l} L C$ mice developed a spontaneous, severe, and uniformly penetrant erosive inflammatory arthritis that was associated with autoantibodies and therefore strongly reminiscent of human rheumatoid arthritis (3). The disease could be transferred by syngeneic bone marrow transplantation and was reversible when $P g g t 1 b^{f l} / f l L C$ mice received wild-type bone marrow, demonstrating that hematopoietic cells, presumably of myeloid lineage, mediate the pathology. Equally striking were the cell biological results. Rather than inactive Rho family proteins, $P g g t 1 b^{f / f l} L C$ macrophages contained RhoA, Rac1, and $\mathrm{Cdc} 42$ proteins that were constitutively loaded with GTP and therefore activated. Nevertheless, Pggt $1 b^{f l / f l} L C$ macrophages showed a striking defect in spreading on tissue culture plastic, a Rho-dependent process that is a hallmark of normal macrophages. Importantly, both Rac1 and RhoA constructs engineered to be prenylated by FTase rescued this defect. Thus, GGTase-I deficiency did affect the actin cytoskeleton in a measurable way. Surprisingly, however, functional studies of motility and phagocytosis showed normal responses. Consistent with the activated Rho proteins, Pggt $1 b^{f / f l} L C$ macrophages secreted substantially more proinflammatory cytokines, such as TNF- $\alpha$,
IL-1 $\beta$, and IL-6, than did Pggt $1 b^{f l /+} L C$ cells. These cytokines are likely pathological, as anti-TNF- $\alpha$ therapy abrogated the arthritis in Pggt $1 b^{f l / f l} L C$ mice. Perhaps most perplexing is the finding that Rac1 was largely membrane associated in Pggt $1 b^{f l / f l} L C$ macrophages.

\section{The role of geranylgeranylation on Rho protein function}

It is not clear how much of the playbook on Rho proteins needs to be rewritten in light of these findings. Rho proteins are regulated by three classes of accessory molecules: guanine nucleotide exchange factors (GEFs), Rho GDP-dissociation inhibitors (RhoGDIs), and Rho GTPase-activating proteins (RhoGAPs). It is believed that prenylation of the GTPase is required for each of these regulatory proteins to exert its effects (Figure 1). First, GEFs activate Rho proteins by catalyzing the exchange of GDP for GTP. Rho GEFs, such as TIAM1 and VAV1, are activated by recruitment to the plasma membrane $(11,12)$, which suggests that only membrane-associated, and therefore prenylated, Rho proteins are substrates for GEFs. This view may have to be revised given the authors' finding that Rho proteins were, if anything, more GTPbound in the absence of prenylation (3).

\section{Figure 1}

Regulation of geranylgeranylated Rho GTPases. Inactive Rho proteins are GDP bound and sequestered in the cytosol by binding to their chaperone RhoGDI, which shields the geranylgeranyl group from the aqueous environment. The activation cycle begins when Rho proteins translocate using their geranylgeranyl lipid moieties to cellular membranes, where they encounter GEFs that catalyze GTP/GDP exchange. GTP-bound Rho proteins engage effectors and are acted on by GAPs that facilitate GTP hydrolysis, returning the Rho proteins to their inactive state that can be extracted from the membrane by free RhoGDI. Central to each step of regulation is the geranylgeranyl lipid. However, in light of the findings of Khan et al., who show that macrophages can function without geranylgeranylation of Rho proteins (3), the role of the lipid modification may have to be reconsidered.
Second, RhoGDI is a cytosolic chaperone for Rho proteins that retains them in an inactive, GDP-bound state. RhoGDI renders prenylated Rho proteins soluble by shielding their lipid moieties (13). Indeed, the cocrystal of Cdc42 with RhoGDI (14) shows a hydrophobic pocket for the lipid and strongly suggests that geranylgeranylation is required for the two proteins to bind. Curiously, Khan et al. found that, although Rac1 and Cdc42 binding to RhoGDI was diminished but not abolished in Pggt $1 b^{f l / f l} L C$ macrophages, RhoA binding to RhoGDI was unaffected (3), suggestive of relatively strong protein-protein interactions in the absence of the protein-lipid interaction. Finally, RhoGAPs are accessory proteins required for hydrolysis of GTP bound to Rho proteins. They are therefore essential negative regulators of Rho signaling. The ability of RhoGAP to regulate Rho proteins has been shown to depend on prenylation of the latter (15). If RhoGAPs are more dependent than are GEFs on geranylgeranylation, it could explain why Khan et al. found Rho GTPases to be constitutively active in GGTase-I-deficient macrophages (3).

How could Rac1 retain membrane association without prenylation? Rac1 has a C-terminal polybasic motif that may be 
sufficient to mediate association with the negatively charged cytoplasmic leaflet of cellular membranes (16). Curiously, in cultured mammalian cells, nonprenylated Rac1 is efficiently transported into the nucleus by virtue of a strong C-terminal nuclear localization signal (17), a finding that Khan et al. could not confirm in Pggt $1 b^{f l / f l} L C$ macrophages (3). Like Rho proteins, Ras is generally considered to require prenylation to function (6) because it too engages its regulatory proteins and effectors at cellular membranes. Interestingly, one recent study found that nonprenylated Ras could support photoreceptor development in flies (18), which indicates that in some contexts, small GTPases can function in the absence of prenylation.

\section{GGTase-I has many substrates}

Rho proteins are not the only important substrates for GGTase-I. Indeed, the human genome encodes 60 proteins that are putative substrates, 25 of which have been validated experimentally (19). Among these are non-GTPases, including enzymes such as 2 ', $3^{\prime}$-cyclic nucleotide $3^{\prime}$-phosphodiesterase and aldehyde dehydrogenase 7 and 8. In addition to Rho family GTPases, the Ras family GTPases Rap and Ral are geranylgeranylated. Strong evidence links Rap1 to inside-out signaling by integrins, including those that mediate the leukocyte adhesion required for cell migration (20). Moreover, Rap1a-null mice have defective macrophages (21). Khan et al.'s observation that Pggt $1 b^{f l / f l} L C$ macrophages can migrate, including into the inflamed peritoneal cavity (3), suggests that integrin activation does not require geranylgeranylated Rap1. Perhaps most provocative, 10 of 12 heterotrimeric $G$ protein $\gamma$ subunits are substrates for GGTase-I (19). Thus, the overwhelming majority of GPCR signaling would appear to require geranylgeranylation. Since normal macrophage function relies on a myriad of GPCRs, the authors' results are all the more perplexing.

\section{Macrophage-driven arthritis and GGTase-I inhibitors}

Autoimmune inflammatory arthritis is thought to be a $\mathrm{T}$ cell-driven disease, with myeloid cells acting as effectors (22). The results of Khan et al. force a reassessment of this paradigm because the evidence for a causative role of macrophages in the pathogenesis of the arthritis observed is unassailable (3). Since macrophages are the most prodigious source of TNF- $\alpha$, which is pathogenic in rheumatoid arthritis, the ability of this type of leukocyte to drive the disease is not without logic. One plausible model might be that, in the absence of GGTase-I, macrophages no longer require signals from $\mathrm{T}$ cells to secrete inflammatory cytokines.

The authors' findings have implications for drug development. The Ras trafficking pathway has long been considered the Achilles' heel of the oncoprotein. FTase inhibitors failed in the clinic because oncogenic K-Ras and N-Ras can escape the blockade by serving as substrates for GGTase-I (23). This has led to an effort to develop GGTase-I inhibitors as anticancer drugs $(24,25)$. If pharmacological inhibition of GGTase-I has the same effects as genetic disruption of Pggt1b, as suggested by some of the data presented by Khan et al. (3), then one might expect such drugs to exhibit significant toxicity in the form of autoimmune disease.

\section{Unanswered questions}

Experimental results often raise more questions than they answer. In the case of unexpected results like those of Khan et al. (3), this is even truer. Why are GGTase-Ideficient myeloid cells so different from fibroblasts, which the authors previously reported underwent a proliferative arrest and failed to migrate (10)? Is geranylgeranylation really dispensable for the function of Rho, Rap, and Ral small GTPases, as well as heterotrimeric $G$ proteins in macrophages? If so, why is geranylgeranylation conserved through evolution? Are alternate membrane-targeting pathways operating in the GGTase-I-deficient macrophages? Why are farnesylated RhoA and Rac1 interchangeable in rescuing the morphological phenotype of $P_{g g t} 1 b^{f l f l} L C$ macrophages, when these proteins have opposite effects on actin remodeling? What proteins lacking geranylgeranylation are responsible for augmented cytokine production, and what is the mechanism? Are lymphocytes, especially Th17 cells, required for the inflammatory arthritis driven by $P g g t 1 b^{f / f l} L C$ macrophages? Do Pggt $1 b^{f l f l} L C$ macrophages inhibit Tregs?

Mouse models are powerful tools. Even when they reveal unexpected results, they offer a ready way to explain them with further study. I expect that the Pggt $1 b^{f l / f l} L C$ mice described here have much more to teach us about the role of prenylation in GTPase function and about the role of macrophages in inflammatory arthritis.

\section{Acknowledgments}

The author thanks Adrienne Cox for insightful discussions. This work was supported by NIH grants GM055279, CA116034, and CA118495 and funding from the Jeffrey Rosenzweig and Children's Tumor Foundations.

Address correspondence to: Mark R. Philips, NYU Cancer Institute, Smilow 1207, 522 First Avenue, New York, New York 10016, USA. Phone: 212.263.7404; Fax: 212.263.9210; E-mail: mark.philips@ nyumc.org.

1. Esteban LM, et al. Targeted genomic disruption of $\mathrm{H}$-ras and $\mathrm{N}$-ras, individually or in combination, reveals the dispensability of both loci for mouse growth and development. Mol Cell Biol. 2001;21(5):1444-1452.

2. Shull MM, et al. Targeted disruption of the mouse transforming growth factor-beta 1 gene results in multifocal inflammatory disease. Nature. 1992;359(6397):693-699.

3. Khan OM, et al. Geranylgeranyltransferase type I (GGTase-I) deficiency hyperactivates macrophages and induces erosive arthritis in mice. J Clin Invest. 2011;121(2):628-639.

4. Wennerberg K, Rossman KL, Der CJ. The Ras superfamily at a glance. J Cell Sci. 2005;118(Pt 5):843-846.

5. Wright LP, Philips MR. Thematic review series: lipid posttranslational modifications. CAAX modification and membrane targeting of Ras.J Lipid Res. 2006;47(5):883-891.

6. Willumsen BM, Cox AD, Solski PA, Der CJ, Buss JE. Novel determinants of H-Ras plasma membrane localization and transformation. Oncogene. 1996;13(9):1901-1909.

7. Allal C, et al. RhoA prenylation is required for promotion of cell growth and transformation and cytoskeleton organization but not for induction of serum response element transcription. J Biol Chem. 2000;275(40):31001-31008.

8. Jaffe AB, Hall A. Rho GTPases: biochemistry and biology. Annu Rev Cell Dev Biol. 2005;21:247-69.

9. Roberts AW, et al. Deficiency of the hematopoietic cell-specific Rho family GTPase Rac2 is characterized by abnormalities in neutrophil function and host defense. Immunity. 1999;10(2):183-196.

10. Sjogren AM, et al. GGTase-I deficiency reduces tumor formation and improves survival in mice with K-RAS-induced lung cancer. J Clin Invest. 2007;117(5):1294-304.

11. Bustelo XR. Regulatory and signaling properties of the Vav family. Mol Cell Biol. 2000;20(5):1461-1477.

12. Michiels F, et al. Regulated membrane localization of Tiam1, mediated by the NH2-terminal pleckstrin homology domain, is required for Rac-dependent membrane ruffling and C-Jun NH2-terminal kinase activation. J Cell Biol. 1997;137(2):387-398.

13. Michaelson D, Silletti J, Murphy G, D’Eustachio P, Rush M, Philips MR. Differential localization of Rho GTPases in live cells. Regulation by hypervariable regions and rhoGDI binding. J Cell Biol. 2001;152(1):111-126.

14. Hoffman GR, Nassar N, Cerione RA. Structure of the Rho family GTP-binding protein Cdc42 in complex with the multifunctional regulator RhoGDI. Cell. 2000;100(3):345-356.

15. Molnar G, Dagher MC, Geiszt M, Settleman J, Ligeti $\mathrm{E}$. Role of prenylation in the interaction of Rho-family small GTPases with GTPase activating proteins. Biochemistry. 2001;40(35):10542-10549.

16. Yeung $\mathrm{T}$, et al. Receptor activation alters inner 
surface potential during phagocytosis. Science. 2006;313(5785):347-351.

17. Michaelson D, et al. Rac1 accumulates in the nucleus during the G2 phase of the cell cycle and promotes cell division. J Cell Biol. 2008;181(3):485-496.

18. Sung PJ, Rodrigues AB, Kleinberger A, Quatela S, Bach EA, Philips MR. Cytosolic ras supports eye development in Drosophila. Mol Cell Biol. 2010;30(24):5649-5657.

19. Reid TS, Terry KL, Casey PJ, Beese LS. Crystallographic analysis of CaaX prenyltransferases com- plexed with substrates defines rules of protein substrate selectivity. J Mol Biol. 2004;343(2):417-433.

20. Bos JL, et al. The role of Rap1 in integrin-mediated cell adhesion. Biochem Soc Trans. 2003;31(pt 1):83-86.

21. Li Y, et al. Rap1a null mice have altered myeloid cell functions suggesting distinct roles for the closely related Rap1a and $1 \mathrm{~b}$ proteins. J Immunol. 2007;179(12):8322-8331.

22. Cope AP, Schulze-Koops H, Aringer M. The central role of T cells in rheumatoid arthritis. Clin Exp Rheumatol. 2007;25(5 suppl 46):S4-S11.
23. Whyte DB, et al. K- and N-Ras are geranylgeranylated in cells treated with farnesyl protein transferase inhibitors. J Biol Chem. 1997;272(22):14459-14464.

24. Kazi A, et al. Blockade of protein geranylgeranylation inhibits Cdk2-dependent p27Kip1 phosphorylation on Thr187 and accumulates p27Kip1 in the nucleus: implications for breast cancer therapy. $\mathrm{Mol}$ Cell Biol. 2009;29(8):2254-2263.

25. Philips MR, Cox AD. Geranylgeranyltransferase I as a target for anti-cancer drugs. J Clin Invest. 2007;117(5):1223-1225.

\title{
Navigational error in the heart leads to premature ventricular excitation
}

\author{
Hiroshi Akazawa and Issei Komuro
}

Department of Cardiovascular Medicine, Osaka University Graduate School of Medicine, Osaka, Japan.

\begin{abstract}
In the normal heart, an insulating barrier separates the atria and ventricles. The only way in which electrical impulses can cross this barrier is via the atrioventricular (AV) node, which delays impulse conduction to ensure the forward flow of the blood. However, in some individuals, additional muscular bundles (accessory pathways) allow rapid conduction of electrical impulses from the atria to the ventricles, resulting in premature ventricular excitation and contraction. In this issue of the JCI, two independent research groups demonstrate that erroneous development of the embryonic AV canal, which performs a similar function to that of the adult $\mathrm{AV}$ node, is a novel mechanism by which accessory pathways can form.
\end{abstract}

Three-dimensional organization of the electrical conduction system allows the heart to generate rhythmic forward flow of the blood (Figure 1A). After triggering atrial contraction, the electrical impulses are delayed at the atrioventricular (AV) junction for an optimal period of time that allows the ventricles to fill up with blood before ventricular contraction is initiated. In adult hearts, this delay is attributable to slow conduction by specialized cardiomyocytes in the AV node. After this delay, the electrical impulses rapidly travel to the ventricular chamber myocardium via the His-Purkinje system and stimulate ventricular contraction. The AV node/His-bundle pathway is the only muscular connection between the atrial and ventricular chambers, which are electrically insulated from each other by the annulus fibrosus, a ring-like struc-

Conflict of interest: Issei Komuro has received research support from Takeda Pharmaceutical Co. Ltd., Daiichi Sankyo Co. Ltd., MSD K.K., Shionogi \& Co. Ltd., Nippon Boehringer Ingelheim Co. Ltd., Astellas Pharma Inc., Novartis Pharma K.K., and Kowa Pharmaceutical Co. Ltd.

Citation for this article: J Clin Invest. 2011; 121(2):513-516. doi:10.1172/JCI46038. ture that consists of connective tissues impermeable to electrical propagation.

Development of the AV conduction system involves a precisely coordinated process of cellular differentiation and multicellular morphogenesis. Therefore, even subtle perturbation of the developmental process can give rise to anatomical or functional defects of the cardiac conduction system and thereby causes serious arrhythmias. For example, in patients with Wolff-Parkinson-White (WPW) syndrome, in addition to the AV node/His-Purkinje system pathway, extra electrically active muscular connections known as accessory pathways exist between the atria and ventricles. Electrical impulses travel via accessory pathways and induce earlier contraction of the ventricular myocardium (preexcitation) than do electrical impulses traveling via the AV node/His-Purkinje system. In this situation, antegrade $\mathrm{AV}$ nodal conduction may immediately trigger retrograde conduction through the accessory pathways and re-excite the atria, leading to initiation and perpetuation of reentrant tachycardia. Even more ominous is the rapid and preexcited ventricular response to atrial fibrillation in patients with WPW syndrome, which can precipitate life-threatening ventricular fibrillation. Despite the clinical importance of ventricular preexcitation, the underlying molecular mechanisms have not been well understood. In this issue of the JCI, two independent research groups report genetic approaches in mice that shed light on the mechanisms for accessory pathway formation and ventricular preexcitation $(1,2)$. Specifically, the data from both groups support the notion that missteps during development of the AV canal give rise to ventricular preexcitation.

\section{Defective formation of the annulus fibrosus}

Molecular insights into the mechanisms underlying ventricular preexcitation were not elucidated until a candidate gene for a familial form of WPW syndrome was identified $(3,4)$. In two unrelated families with WPW syndrome accompanied by hypertrophic cardiomyopathy, a dominant missense mutation (R302Q) was observed in the gene that encodes the $\gamma 2$ regulatory subunit of AMP-activated protein kinase (AMPK), PRKAG2 $(3,4)$. Subsequent biochemical and histological studies indicated that PRKAG2 mutations affect the basal activity of AMPK, a "fuel gauge" participating in the regulation of energy homeostasis (5). Transgenic mice overexpressing human PRKAG2 with a WPW syndrome-causing mutation, such as R302Q or N488I, in the heart, showed cardiac glycogen storage and manifested ventricular hypertrophy and preexcitation $(6,7)$. Strikingly, histopathology of the hearts revealed that the annulus fibrosus was disrupted by intercalation of glycogen-loaded cardiomyocytes. These studies have impli- 